\title{
Periodontal bacteria and cardiovascular problems
}

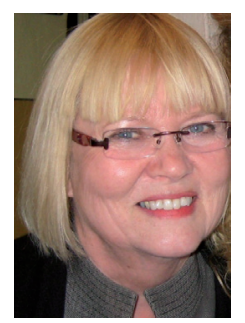

“...in periodontitis the enduring pathology ... forms

a biological basis for systemic effects

related to the development

of atherosclerosis and its complications."

\section{Sirkka E Asikainen}

Section of Oral Microbiology, Umea University, SE-90187 Umea, Sweden a Tel.: +46907 856166

= Fax: +46907 856053 m sirkka.asikainen@odont.umu.se

The Finnish studies that linked dental infections to cardiovascular diseases $[1,2]$ received widespread interest at the end of the $1980 \mathrm{~s}$ and kindled great enthusiasm among dental researchers. In addition to the keen scientific interest, a new possibility was also seen to raise the status of dental infections among human infectious diseases and to increase public awareness of dental diseases as infections. The concept that atherosclerosis is an inflammatory disease $[3,4]$ further enhanced research interest in dental infections and particularly in periodontitis as possible inflammatory triggers. After two decades, the controversial results from epidemiological studies [5] have gradually raised doubts of the validity of the suggested association between periodontitis and cardiovascular diseases. By contrast, the fact remains that in untreated or undertreated periodontitis the persistent bacterial growth on tooth surfaces and chronic inflammation in surrounding periodontal tissues provide a biologically feasible basis for inducing systemic effects that are considered to increase the risk of cardiovascular pathology.

The systemic inflammatory effect of periodontitis has been studied by measuring levels of surrogate biomarkers for inflammation, such as proinflammatory cytokines and acute phase proteins, especially C-reactive protein [6]. These levels seem to be elevated in periodontitis patients in comparison to periodontally healthy subjects and the levels tend to decrease after treatment of periodontitis [7]. However, the inflammatory response is nonspecific by nature and can be induced by various stimuli. For example, coexisting but undiagnosed silent or acute infections and other pathological conditions and/or their treatment have their effects on patient's inflammatory status. Thus, the relative contribution of periodontitis, or of any other specified infection, to systemic inflammation remains difficult to determine.
Antibody reaction may serve as a more specific approach than inflammatory biomarkers for determining systemic host response to periodontitis infection. Along this line of thinking, serum antibody levels were measured against selected periodontitis-associated species and a positive association was found between periodontitis and cardiovascular disease [8] for the first time on a biological basis. However, periodontitis is a multibacterial infection and even though the two bacterial species were selected according to culture-based knowledge and after validation in patients with and without periodontitis [9], it can today be discussed whether relevant bacterial species specific to periodontitis were/are used in corresponding immunoassays.

Culture-independent nucleic acid-based techniques, particularly $16 \mathrm{~S}$ rRNA gene sequencing approaches, have recently made it possible to detect and identify a variety of previously unknown bacteria from subgingival samples. At present, approximately 700 phylotypes are known [10], of the more than 1000 estimated phylotypes. Currently available applications that utilize the acquired sequence data include DNA microarrays that provide simultaneous and rapid detection of several hundred cultivable and not-yet-cultured bacterial species/phylotypes. Quantitative real-time PCR allows prompt discovery of the quantity and proportions of selected species in complex subgingival samples. In addition, the rapid development of sequencing technology makes faster and faster sequence data collection possible. Although handling and interpretation of the huge amount of data are key issues that first need to be resolved, thereafter, new models for periodontal microbiota may be constructed on, for example, the basis of bacterial community profiling.

Besides the technological advances in molecular biology, another driving force revolutionizing our knowledge of periodontal infections

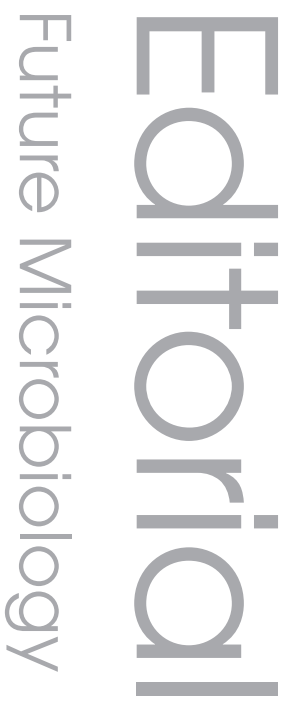

66

....periodontitis is a multibacterial infection ... 700 phylotypes are known."

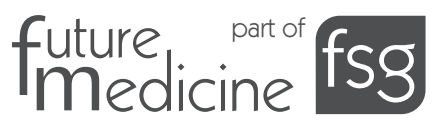




\section{“...another driving force revolutionizing our knowledge of periodontal infections is the biofilm science."}

is the biofilm science. Despite being pioneered in environmental microbiology several decades ago, it was only relatively recently launched to medicine $[11,12]$. It is obvious that the biofilm concept is changing the view of chronic infections that were previously strongly influenced by concepts on acute infections. Compared with other biofilm-associated infections the complexity of subgingival microbial biofilm represents a challenge. Taking on this challenge seems necessary, since the current knowledge of the health impact of subgingival microbiota is mainly based on studies comparing clinical periodontal status to bacterial culture or PCR detection of target bacteria from subgingival samples or on studies using pure cultures of selected bacterial species in a variety of experimental models. By contrast, little is understood of the community life style of subgingival microbiota. The good news today is that the biofilm science connects dental infections to a wider biological context provided by environmental and medical microbiology.

For the time being, culture results still retain their historical position as the conceptual basis in periodontal microbiology. Extensive efforts have revealed that no single bacterium can be regarded as the particular periodontal pathogen for all patients. It appears that different species belonging to the cultured indigenous oral microbiota can overgrow in the complex subgingival bacterial community in different patients and even at different subgingival sites or time points in the same patient. Despite this, culture-dependent studies provide evidence for shared characteristics among the microbiota in periodontitis when compared with the microbiota in periodontally healthy individuals. They also pinpoint certain species as possible periodontal pathogens [13]. These species include mainly Gram-negative bacteria, such as Aggregatibacter (previously Actinobacillus) actinomycetemcomitans, Porphyromonas gingivalis, Tannerella forsythia, Prevotella intermedia, and Campylobacter rectus. Consistent with the specific-pathogen concept, periodontal microbiology research during the last decades has mainly focused on culture and PCR detection of these individual species from clinical samples in a variety of study setups. In mechanistic studies, bacteria such as $P$. gingivalis and $A$. actinomycetemcomitans have been commonly used as model species to investigate, for example, how periodontal bacteria could contribute to the development of atherosclerosis and its complications.
The new methods to detect and identify subgingival bacteria independent of culture methods have already revealed results that shake the traditional grounds in this field. For example, the predominant bacteria or bacterial groups found in periodontitis [14] are not the same periodontitis-associated bacteria identified in hundreds of culture-based studies during the past 30 years. Questions are raised as to whether culture-dependent methods bias our understanding of periodontal microbiology and whether they have directed our attention to irrelevant bacteria. If so, there may be consequences for the prevailing concept of the specific role of the aforementioned bacterial species in the etiology and progression of periodontitis, as well as for the validity of using these bacteria as model species of periodontal pathogens.

Subgingivally colonizing bacteria live on tooth surfaces outside the epithelial lining of the body. How could these bacteria contribute to a systemic inflammatory state in periodontitis patients? The traditional explanation has been that bacteremic episodes occur during their everyday activities, such as mastication and tooth brushing. Interestingly, bacteria can only be cultured from blood samples for a brief time period after the oral stimulation is discontinued, indicating that bacteria are efficiently cleared from circulation. That the translocated bacteria are capable of colonizing distant body sites is supported by the occasional occurrence of severe nonoral infections, such as endocarditis, brain abscesses and other types of infections, caused by these bacteria [15].

The main route for subgingival bacteria to enter the parenteral space is probably through microulcerations in nonkeratinized crevicular epithelium facing the tooth surface. Ulcerations already appear during the early phases of periodontal inflammation and they continuously break the integrity of crevicular epithelium in the course of periodontitis progression. After the bacteria have gained access to the abundant blood and lymph vessel networks in the inflamed periodontal tissues the route to larger neck vessels and via the superior vena cava to the heart is straightforward. With every heartbeat periodontal bacteria can then be spread to the pulmonary and systemic circulatory systems. Still, it is surprising that nonoral infections caused by periodontitis-associated bacteria are infrequent, even despite the high prevalence of periodontitis in adult populations.

Viable oral or nonoral bacteria have hardly ever been found in atheroma plaques, whereas the recovery of bacterial DNA is common. Since this DNA originates from a variety of nonoral and oral bacteria [16], it seems unlikely that only 
certain bacterial species or groups would be associated with atherogenesis. Rather it may mean, if the concept is accepted, that many species have the potential to induce host responses that predispose to atheroma development. Thus, instead of restricting our attention to bacteremia or certain bacterial species or specified infections, an approach considering bacteria-associated molecular patterns (BAMPs) [17] as a basic unit opens new possibilities for mechanistic studies on atherogenesis in chronic infections. The BAMPs are independent of bacterial viability or species and their significance as inflammatory stimulators is well known. They are established ligands for Toll-like receptors [18] and ligand binding initiates signaling that leads to the upregulation of gene expression in the key cells of atherogenesis, for example adhesion molecules in endothelial cells and proinflammatory cytokines and chemokines in monocytes/macrophages and lymphocytes.

Even though the pathogenic potential of BAMPs as individual bacterial cell components is widely discussed in the literature, little is known of the mechanisms by which they are delivered in biofilm-associated chronic infections. Aside from the fact that lysed bacteria liberate bacterial material, the release of BAMPs from live biofilms provides an interesting scenario for periodontitis-associated systemic effects. While releasing outer membrane vesicles that serve as delivery packages for membrane-bound BAMPs, live Gram-negative periodontal bacteria can also release free-soluble BAMPs independently of vesicles [19]. The released free-soluble BAMPs from both biofilm and planktonic life-forms of Gram-negative bacteria have been shown to induce an upregulation of gene and protein expression of a variety of proinflammatory cytokines in the human whole blood [20]. Based on this early experimental data it is tempting to speculate that the release of BAMPs from subgingival biofilms could provide an important mechanism by which chronic periodontal inflammation is perpetuated and systemic dissemination of bacterial material sustained. In free-soluble form, the BAMPs may readily gain access from the subgingival space into blood/lymph microvasculature in adjacent periodontal tissues, which opens the route for their systemic spread. In blood circulation and arterial intima they may induce proinflammatory and proatherogenic host responses that promote endothelial dysfunction and atherothrombogenesis.

In periodontitis, the environment for bacterial growth is unique. Nowhere else in the body is the epithelial lining penetrated by calcified tissue that provides a solid, nonregenerating surface for bacterial colonization and biofilm formation. An evoked host defense cannot remove bacterial biofilms from the subgingival tooth surfaces. It can, however, gradually segregate teeth from alveolar bone and thus get rid of the tenacious biofilms. From the whole-body perspective, the final outcome, loss of affected teeth, can be regarded as a successful intrinsic anti-infection strategy. Most commonly, periodontal tissue destruction is, however, slow and the affected teeth may only finally loosen after decades. That for most of its progression time periodontitis is silent and painless and, therefore, easily unnoticed, does not diminish the significance of the concurrent immunoinflammatory host reactions.

When considering that periodontitis in its early phase is easily diagnosed and inexpensively treated, it is surprising that periodontitis is still widespread in middle-aged and older populations [21,22]. This situation is unexpected particularly in the USA and Europe, where a massive number of periodontists and dental hygienists have been educated and information of periodontal disease has been actively spread to the general public during the past 30 years. A treatment strategy that involves replacement of periodontitis-affected teeth with dental implants has, however, exponentially increased in dentate patients during recent decades. The dilemma is that these patients have proven their susceptibility to periodontal tissue destruction. In addition, similar to the natural teeth, dental implants also penetrate the oral epithelium and provide solid surfaces for bacterial biofilm growth. As expected, the composition of bacterial biofilms [23] and the inflammatory response in implant-surrounding tissues [24] closely resembles those around natural teeth. An analysis of the literature showed that exposure of dental implants to the oral environment for at least five years led to peri-implantitis more frequently than in half of the treated subjects [25]. Furthermore, like periodontitis, peri-implantitis also provides subgingival biofilms and inflammatory response in surrounding tissues, which can induce systemic effects.

The controversial results on the association of periodontitis and atherosclerosis-related cardiovascular diseases have gradually raised doubts about the validity of the suggested association. Still, in periodontitis the enduring pathology involving persistent bacterial infection and chronic inflammation forms a biological basis for systemic effects related to the development of atherosclerosis and its complications. But,

"That for most of its
progression time
periodontitis is silent and
painless ... does not
diminish the significance
of the concurrent
immunoinflammatory
host reactions."


obtaining proof for a causal relationship between periodontitis and cardiovascular diseases can be expected to be difficult. One of the main reasons is that both diseases are chronic and progress gradually and asymptomatically over decades. Expectations for clear-cut results may be a remnant of the concepts and knowledge concerning diseases caused by acute single-pathogen infections. Other approaches may be needed for chronic infections associated with complex bacterial communities.

New concepts, such as the community lifestyle of subgingival microbiota, and new molecular biological tools, such as metagenomics, are available for a fresh start in periodontal research. Analysis of bacterial genes (gene-centric approach) instead of bacteria in subgingival bacterial communities and a better understanding of the pathogenic mechanisms of these communities will provide exciting local and systemic corollaries.

\section{Financial \& competing interests disclosure \\ The author has no relevant affiliations or financial involvement with any organization or entity with a financial interest in or financial conflict with the sub- ject matter or materials discussed in the manuscript. This includes employment, consultancies, honoraria, stock ownership or options, expert testimony, grants or patents received or pending, or royalties. \\ No writing assistance was utilized in the production of this manuscript.}

\section{Bibliography}

1. Mattila KJ, Nieminen MS, Valtonen VV et al.: Association between dental health and acute myocardial infarction. BMJ 298, 779-781 (1989).

2. Syrjanen J, Peltola J, Valtonen V et al.: Dental infections in association with cerebral infarction in young and middleaged men. J. Intern. Med. 225, 179-184 (1989).

3. Ross R: Atherosclerosis is an inflammatory disease. Am. Heart J. 138, S419-S420 (1999).

4. Libby P: Inflammation in atherosclerosis. Nature 420, 868-874 (2002).

5. Beck JD, Offenbacher S: Systemic effects of periodontitis: epidemiology of periodontal disease and cardiovascular disease. J. Periodontol. 76, 2089-2100 (2005).

6. Willerson JT, Ridker PM: Inflammation as a cardiovascular risk factor. Circulation 109, 2-10 (2004).

7. Paraskevas S, Huizinga JD, Loos BG: A systematic review and meta-analyses on C-reactive protein in relation to periodontitis. J. Clin. Periodontol. 35, 277-290 (2008).

8. Pussinen PJ, Jousilahti P, Alfthan G et al.: Antibodies to periodontal pathogens are associated with coronary heart disease. Arterioscler. Thromb. Vasc. Biol. 23, 1250-1254 (2003).

9. Pussinen PJ, Vilkuna-Rautiainen T, Alfthan G, Mattila K, Asikainen S: Multiserotype enzyme-linked immunosorbent assay as a diagnostic aid for periodontitis in large-scale studies. J. Clin. Microbiol. 40, 512-518 (2002).
10. Paster BJ, Olsen I, Aas JA, Dewhirst FE: The breadth of bacterial diversity in the human periodontal pocket and other oral sites. Periodontol. 2000 42, 80-87 (2006).

11. Costerton JW, Stewart PS, Greenberg EP: Bacterial biofilms: a common cause of persistent infections. Science 284, 1318-1322 (1999).

12. del Pozo JL, Patel R: The challenge of treating biofilm-associated bacterial infections. Clin. Pharmacol. Ther. 82, 204-209 (2007).

13. Socransky SS, Haffajee AD: The bacterial etiology of destructive periodontal disease: current concepts. J. Periodontol. 63, 322-331 (1992).

14. Kumar PS, Griffen AL, Moeschberger ML, Leys EJ: Identification of candidate periodontal pathogens and beneficial species by quantitative $16 \mathrm{~S}$ clonal analysis. J. Clin. Microbiol. 43, 3944-3955 (2005).

15. Gendron R, Grenier D, Maheu-Robert L: The oral cavity as a reservoir of bacterial pathogens for focal infections. Microbes Infect. 2, 897-906 (2000).

16. Ott SJ, El Mokhtari NE, Musfeldt M et al.: Detection of diverse bacterial signatures in atherosclerotic lesions of patients with coronary heart disease. Circulation 113, 929-937 (2006).

17. Erridge C: The roles of pathogen-associated molecular patterns in atherosclerosis. Trends Cardiovasc. Med. 18, 52-56 (2008).

18. Akira S, Takeda K, Kaisho T: Toll-like receptors: critical proteins linking innate and acquired immunity. Nat. Immunol. 2, 675-680 (2001).
19. Karched M, Ihalin R, Eneslatt $\mathrm{K}$ et al.: Vesicle-independent extracellular release of a proinflammatory outer membrane lipoprotein in free-soluble form. BMC Microbiol. 8, 18 (2008).

20. Oscarsson J, Karched M, Thay B, Chen C, Asikainen S: Proinflammatory effect in whole blood by free soluble bacterial components released from planktonic and biofilm cells. BMC Microbiol. 8, 206 (2008).

21. Baelum V, van Palenstein Helderman W, Hugoson A, Yee R, Fejerskov O: A global perspective on changes in the burden of caries and periodontitis: implications for dentistry. J. Oral Rehabil. 34, 872-906 (2007).

22. Boehm TK, Scannapieco FA: The epidemiology, consequences and management of periodontal disease in older adults. J. Am. Dent. Assoc. 138(Suppl. 1), S26-S33 (2007).

23. Mombelli A: Microbiology and antimicrobial therapy of peri-implantitis. Periodontol. 2000 28, 177-189 (2002).

24. Lindhe J, Berglundh T: The interface between the mucosa and the implant. Periodontol. 2000 17, 47-54 (1998).

25. Zitzmann NU, Berglundh T: Definition and prevalence of peri-implant diseases. J. Clin. Periodontol. 35, 286-291 (2008).

\section{Affiliation}

- Sirkka Asikainen, PhD, DDS Professor and Chairman, Section of Oral Microbiology, Umea University, SE-90187 Umea, Sweden Tel.: +46907856 166; Fax: +46907 856053 ; sirkka.asikainen@odont.umu.se 